\title{
PAIRS OF CLIFFORD ALGEBRAS OF THE HURWITZ TYPE
}

\author{
WIESłAW KRÓLIKOWSKI \\ Institute of Mathematics, Polish Academy of Sciences, Eódź Branch \\ Narutowicza 56, 90-136 Eódź, Poland
}

\begin{abstract}
For a given Hurwitz pair $\left[S\left(Q_{S}\right), V\left(Q_{V}\right)\right.$, o] the existence of a bilinear mapping * : $C\left(Q_{S}\right) \times C\left(Q_{V}\right) \rightarrow C\left(Q_{V}\right)$ (where $C\left(Q_{S}\right)$ and $C\left(Q_{V}\right)$ denote the Clifford algebras of the quadratic forms $Q_{S}$ and $Q_{V}$, respectively) generated by the Hurwitz multiplication "o" is proved and the counterpart of the Hurwitz condition on the Clifford algebra level is found. Moreover, a necessary and sufficient condition for " $\star$ " to be generated by the Hurwitz multiplication is shown.
\end{abstract}

1. Introduction. The general Hurwitz problem was studied e.g. by Ławrynowicz and Rembieliński [2-4]. They introduced the notions of "Hurwitz pairs" and "pseudoHurwitz pairs" and gave their systematic classification according to the relationship with real Clifford algebras. In the present work we show the existence of a bilinear mapping $\star: C\left(Q_{S}\right) \times C\left(Q_{V}\right) \rightarrow C\left(Q_{V}\right)$, where $(S, V, \circ)$ is a given Hurwitz pair which makes the following diagram:

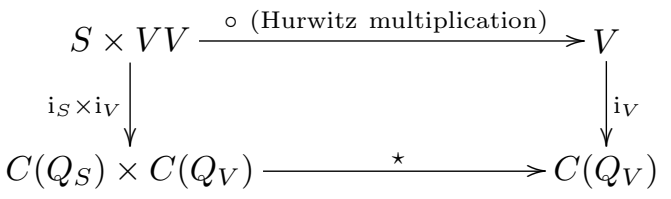

commutative.

Moreover, we prove that if such a mapping exists and satisfies the following "algebraic Hurwitz condition": $N\left(x_{S} \star y_{V}\right)=N\left(x_{S}\right) N\left(y_{V}\right)$ for any $x_{S} \in \Gamma_{S}$ and $y_{V} \in \Gamma_{V}$, where $\Gamma$ denotes the Clifford group of the Clifford algebra $C(Q)$ and $N$ is a spinor norm, then $\star$ is generated by the Hurwitz multiplication, i.e. $\star_{\mid S \times V}=0$. An example of a mapping $\star$ which does not satisfy the $N$-norm condition is given. Since in the meantime the detailed proofs have appeared in [1], they are only sketched here.

1991 Mathematics Subject Classification: Primary 15A66, Secondary 30G35.

Research supported by the KBN grant PB 211409101.

The paper is in final form and no version of it will be published elsewhere. 
2. Product of Clifford algebras generated by the Hurwitz multiplication. Let $(S, V, \circ)$ be a Hurwitz pair. Suppose that the vector spaces $S$ and $V$ are equipped with non-degenerate quadratic forms $Q_{S}$ and $Q_{V}$, respectively. We will only consider the elliptic and hyperbolic cases (see, e.g. [2-4]). In $S$ and $V$ we choose some bases $\left(\epsilon_{\alpha}\right)$ and $\left(e_{j}\right)$ with $\alpha=1, \ldots, p=\operatorname{dim} S ; j=1, \ldots, n=\operatorname{dim} V$. Assume that $p \leq n$.

Let $C\left(Q_{S}\right)$ (resp. $\left.C\left(Q_{V}\right)\right)$ denote the Clifford algebra of $\left(S, Q_{S}\right)$ (resp. $\left(V, Q_{V}\right)$ ). There are canonical injections $i_{S}: S \rightarrow C\left(Q_{S}\right)$ and $i_{V}: V \rightarrow C\left(Q_{V}\right)$. Then we get the diagram (2). It would be interesting to complete the diagram (2) by the suitable mapping $C\left(Q_{S}\right) \times C\left(Q_{V}\right) \rightarrow C\left(Q_{V}\right)$. Define the following mapping $\star: C\left(Q_{S}\right) \times C\left(Q_{V}\right) \rightarrow C\left(Q_{V}\right)$ by:

$$
\left\{\begin{array}{l}
1_{S} \star y_{V}:=y_{V}, \\
\left(\epsilon_{i_{1}} \ldots \epsilon_{i_{r}}\right) \star\left(e_{j_{1}} \ldots e_{j_{k}}\right):= \begin{cases}e_{j_{k}} \ldots e_{j_{r+1}}\left(\epsilon_{i_{r}} \circ e_{j_{r}}\right) \ldots\left(\epsilon_{i_{1}} \circ e_{j_{1}}\right), & r<k \\
\left(\epsilon_{i_{r}} \circ e_{j_{r}}\right) \ldots\left(\epsilon_{i_{1}} \circ e_{j_{1}}\right), & r=k, \\
\epsilon_{i_{r}} \circ\left[\epsilon _ { i _ { r - 1 } } \circ \left[\ldots \circ \left[\epsilon_{i_{k+1}}\right.\right.\right. \\
\left.\circ\left[\left(\epsilon_{i_{k}} \circ e_{j_{k}}\right) \ldots\left(\epsilon_{i_{1}} \circ e_{j_{1}}\right)\right] \ldots\right],\end{cases} \\
\left(\epsilon_{i_{1}} \ldots \epsilon_{i_{r}}\right) \star 1_{V}:=\left\|\epsilon_{i_{1}}\right\| \ldots\left\|\epsilon_{i_{r}}\right\| 1_{V}
\end{array}\right.
$$

for $1 \leq r \leq p, 1 \leq i_{1}<\ldots<i_{r} \leq p ; 1 \leq k \leq n, 1 \leq j_{1}<\ldots<j_{k} \leq n$. Then, the required mapping $\star: C\left(Q_{S}\right) \times C\left(Q_{V}\right) \rightarrow C\left(Q_{V}\right)$ is defined by the bilinear extension of (2).

Remark. If $\left(S, Q_{S}\right)$ is a Euclidean vector space then all $\left\|\epsilon_{i}\right\|^{2}>0$. In this case the Clifford algebras $C\left(Q_{S}\right)$ and $C\left(Q_{V}\right)$ are considered to be real. But, if $\left(S, Q_{S}\right)$ is a pseudo-Euclidean vector space then there are some $\epsilon_{i_{1}}, \ldots, \epsilon_{i_{r}}, 1 \leq r \leq p$, such that $\left\|\epsilon_{i_{s}}\right\|^{2}<0,1 \leq s \leq r$. This time the Clifford algebras have to be treated as complex ones.

Proposition. $\star$ is a well defined bilinear mapping. Moreover, $\star_{\mid S \times V}=0$, the Hurwitz multiplication, i.e. the diagram (1) is commutative.

LEMmA. Let $x_{S} \in \Gamma_{S}$ and $y_{V} \in \Gamma_{V}$, where $\Gamma_{S}$ (resp. $\Gamma_{V}$ ) denotes the Clifford group in $C\left(Q_{S}\right)$ (resp. $C\left(Q_{V}\right)$ ) and let $N_{S}, N_{V}$ be the spinor norms in $C\left(Q_{S}\right)$ and $C\left(Q_{V}\right)$, respectively. Then

$$
N_{V}\left(x_{S} \star y_{V}\right)=N_{S}\left(x_{S}\right) N_{V}\left(y_{V}\right) .
$$

TheOREM. Let $S$ and $V$ be real vector spaces equipped with non-degenerate quadratic forms $Q_{S}$ and $Q_{V}$, respectively. Denote by $C^{\mathbb{C}}\left(Q_{S}\right)\left(\right.$ resp. $\left.C^{\mathbb{C}}\left(Q_{V}\right)\right)$ the complex Clifford algebras of $\left(S, Q_{S}\right)$ (resp. $\left.\left(V, Q_{V}\right)\right)$. Suppose that there is a bilinear mapping $\star: C^{\mathbb{C}}\left(Q_{S}\right) \times$ $C^{\mathbb{C}}\left(Q_{V}\right) \rightarrow C^{\mathbb{C}}\left(Q_{V}\right)$ satisfying the condition (3). Then $\star$ is generated by the Hurwitz multiplication, i.e. $\star_{\mid S \times V}=\circ$, where $\circ: S \times V \rightarrow V$ is a bilinear mapping such that $\|s \circ v\|_{V}=\|s\|_{S}\|v\|_{V}$ for all $s \in S$ and $v \in V$.

Proof. Let $s \in S \subset \Gamma_{S}$ and $v \in V \subset \Gamma_{V}$. By definition of $N$ we have

$$
N_{V}(s \star v)=N_{S}(s) N_{V}(v)=\|s\|_{S}^{2}\|v\|_{V}^{2} \in \mathbb{R} .
$$

Let $\left(e_{1}, \ldots, e_{n}\right)$ be an orthogonal base in $V$. Suppose

$$
s \star v=a_{0}+\sum_{i=1}^{n} a^{i} e_{i}+\sum_{l=2}^{n} \sum_{i_{1}<\ldots<i_{l}} a_{l}^{{ }^{i_{1}} \ldots i_{l}} e_{i_{1}} \ldots e_{i_{l}} .
$$


Then

$N(s \star v)=a_{0}^{2}+\sum_{i=1}^{n}\left(a^{i}\right)^{2} Q_{V}\left(e_{i}\right)+\sum_{l=2}^{n} \sum_{i_{1}<. .<i_{l}}\left(a_{l}^{i_{1} . . i_{l}}\right)^{2} Q_{V}\left(e_{i_{1}}\right) \ldots Q_{V}\left(e_{i_{l}}\right)+R\left(e_{1}, \ldots, e_{n}\right)$,

where

$$
R\left(e_{1}, \ldots, e_{n}\right)=\sum b^{i} e_{i}+\sum_{i<j} b^{i j} e_{i} e_{j}+\ldots+\sum_{i_{1}<\ldots<i_{m}} b^{i_{1} \ldots i_{m}} e_{i_{1}} \ldots e_{i_{m}}+b e_{1} \ldots e_{n} .
$$

Since $N(s \star v)$ is a scalar then $R\left(e_{1}, \ldots, e_{n}\right)$ must vanish. The multiplication $\star$ is bilinear so the coefficients $a_{0}, a^{i}$ and $a_{l}^{i_{1} \ldots i_{l}}$ are bilinear functions in $s$ and $v$. Thus $N(s \star v)$ should be separated into two parts, first depending only on $s$ and second only on $v$. Then we can write

$$
\begin{aligned}
a_{0}^{2}+\sum_{i=1}^{n}\left(a^{i}\right)^{2} Q_{V}\left(e_{i}\right)+\sum_{l=2}^{n} \sum_{i_{1}<\ldots<i_{l}}\left(a_{l}^{i_{1} \ldots i_{l}}\right)^{2} Q_{V}\left(e_{i_{1}}\right) \ldots Q_{V}\left(e_{i_{l}}\right) \\
=\|s\|_{S}^{2}\|v\|_{V}^{2} \\
\quad=\|s\|_{S}^{2}\left[c_{0}^{2}+\sum_{i=1}^{n}\left(c^{i}\right)^{2} Q_{V}\left(e_{i}\right)+\sum_{l=2}^{n} \sum_{i_{1}<\ldots<i_{l}}\left(c_{l}^{i_{1} \ldots i_{l}}{ }^{2} Q_{V}\left(e_{i_{1}}\right) \ldots Q_{V}\left(e_{i_{l}}\right)\right]\right.
\end{aligned}
$$

Thus, the following equality has to be satisfied:

$$
c_{0}^{2}+\sum_{i=1}^{n}\left(c^{i}\right)^{2} Q_{V}\left(e_{i}\right)+\sum_{l=2}^{n} \sum_{i_{1}<\ldots<i_{l}}\left(c_{l}^{i_{1} \ldots i_{l}}\right)^{2} Q_{V}\left(e_{i_{1}}\right) \ldots Q_{V}\left(e_{i_{l}}\right)=\sum_{i=1}^{n}\left(v^{i}\right)^{2} Q_{V}\left(e_{i}\right) .
$$

The coefficients $c_{0}, c^{i}, c_{l}^{i_{1} \ldots i_{l}}$ are linear in $v$ so, by continuity, we can write

$$
c_{0}(v)=c_{0 j} v^{j}, \quad c^{i}(v)=c_{j}^{i} v^{j}, \quad c_{l}^{i_{1} \ldots i_{l}}(v)=c_{l j}^{i_{1} \ldots i_{l}} v^{j} .
$$

Thus, for any $1 \leq j, k \leq n$ we get the identity

$$
c_{0 j} c_{0 k}+\sum_{i=1}^{n}\left(c_{j}^{i} c_{k}^{i}-\delta_{j}^{i} \delta_{k}^{i}\right) Q_{V}\left(e_{i}\right)+\sum_{l=2}^{n} \sum_{i_{1}<\ldots<i_{l}} c_{l j}^{i_{1} \ldots i_{l}} c_{l k}^{i_{1} \ldots i_{l}} Q_{V}\left(e_{i_{1}}\right) \ldots Q_{V}\left(e_{i_{l}}\right) \equiv 0 .
$$

Take an orthogonal transformation $R \in O\left(Q_{V}\right)$. In a new base $e^{\prime}=R e$ we have

$$
c_{0 j} c_{0 k}+\sum_{i=1}^{n}\left(\widetilde{c}_{j}^{i} \widetilde{c}_{k}^{i}-\delta_{j}^{i} \delta_{k}^{i}\right) Q_{V}\left(R e_{i}\right)+\sum_{l=2}^{n} \sum_{i_{1}<\ldots<i_{l}} \widetilde{c}_{l j}^{i_{1} \ldots i_{l}} \widetilde{c}_{l k}^{i_{1} \ldots i_{l}} Q_{V}\left(R e_{i_{1}}\right) \ldots Q_{V}\left(R e_{i_{l}}\right) \equiv 0 .
$$

But $Q_{V}\left(R e_{i}\right)=Q_{V}\left(e_{i}\right)$. Then the new coefficients $\widetilde{c}_{j}$ and $\widetilde{c}_{l j}^{i_{1} \ldots i_{l}}$, obtained by the changing of the base, satisfy the same identity as the previous ones. This is possible if and only if

$$
\begin{aligned}
& c_{0 j} \equiv 0 \quad \text { for } j=1, \ldots, n, \\
& c_{j}^{i} c_{k}^{i}-\delta_{j}^{i} \delta_{k}^{i} \equiv 0 \quad \text { for } 1 \leq i, j, k \leq n \\
& c_{l j}^{i_{1} \ldots i_{l}} \equiv 0 \quad \text { for } l=2, \ldots, n ; 1 \leq i_{1}<\ldots<i_{l} \leq n ; j=1, \ldots, n .
\end{aligned}
$$

Thus, we get $s \star v=\|s\|_{S} \sum_{i=1, j=1}^{n} c_{j}^{i} v^{j} e_{i} \in V$ and $\|s\|_{S}^{2}\|v\|_{V}^{2}=N_{V}(s \star v)=\|s \star v\|_{V}^{2}$, so $\star_{\mid S \times V}$ satisfies the Hurwitz condition, as required.

ExAmple. We now construct a bilinear map $\square: C^{\mathbb{C}}\left(Q_{S}\right) \times C^{\mathbb{C}}\left(Q_{V}\right) \rightarrow C^{\mathbb{C}}\left(Q_{V}\right)$ which does not satisfy the condition (4). Choose some bases $\left(\epsilon_{\alpha}\right)$ and $\left(e_{j}\right)$ in $S$ and $V$, 
respectively. Define

$$
\left\{\begin{aligned}
1_{S} \square 1_{V} & :=e_{1} \ldots e_{n} \\
1_{S} \square\left(e_{i_{1}} \ldots e_{i_{k}}\right) & :=e_{i_{1}} \widehat{\ldots} e_{i_{k}} \\
1_{S} \square\left(e_{1} \ldots e_{n}\right) & :=1_{V} \\
\left(\epsilon_{j_{1}} \ldots \epsilon_{j_{r}}\right) \square\left(e_{i_{1}} \ldots e_{i_{k}}\right) & :=\left\|\epsilon_{j_{1}}\right\| \ldots\left\|\epsilon_{j_{r}}\right\| e_{i_{1}} \ldots e_{i_{k}}, \\
\left(\epsilon_{j_{1}} \ldots \epsilon_{j_{r}}\right) \square 1_{V} & :=\left\|\epsilon_{j_{1}}\right\| \ldots\left\|\epsilon_{j_{r}}\right\| e_{1} \ldots e_{n}, \\
\left(\epsilon_{j_{1}} \ldots \epsilon_{j_{r}} \square\left(e_{1} \ldots e_{n}\right)\right. & :=\left\|\epsilon_{j_{1}}\right\| \ldots\left\|\epsilon_{j_{r}}\right\| 1_{V}
\end{aligned}\right.
$$

where "?" is defined by

$$
e_{i_{1}} \widehat{\ldots} e_{i_{r}}:=e_{j_{1}} \ldots e_{j_{s}} \text { with } j_{1}<\ldots<j_{s} \text { and }\left(i_{1}, \ldots, i_{r}, j_{1}, \ldots, j_{s}\right)=(1, \ldots, n) .
$$

The map $\square: C^{\mathbb{C}}\left(Q_{S}\right) \times C^{\mathbb{C}}\left(Q_{V}\right) \rightarrow C^{\mathbb{C}}\left(Q_{V}\right)$ is defined by the bilinear extension of (5). It is easy to see that $\square$ does not satisfy the condition (4). Indeed, take $s \in S$ and $v \in V$. We have

$$
s \square v=s^{\alpha} v^{i} \epsilon_{\alpha} \square e_{i}=s^{\alpha} v^{i}\left\|\epsilon_{\alpha}\right\|_{S} e_{1} \ldots \widehat{e}_{i} \ldots e_{n} \notin V .
$$

and

$$
\left.N_{V}(s \square v)=s^{\alpha} s^{\beta}\left\|\epsilon_{\alpha}\right\|_{S}\left\|\epsilon_{\beta}\right\|_{S}\left(v^{i}\right)^{2} Q_{V}\left(e_{1}\right) \ldots \widehat{Q_{V}\left(e_{i}\right.}\right) \ldots O_{V}\left(e_{n}\right) .
$$

Suppose that $N_{V}(s \square v)=N_{S}(s) N_{V}(v)$. Then we get

$$
\begin{aligned}
\sum_{\alpha, \beta} s^{\alpha} s^{\beta}\left\|\epsilon_{\alpha}\right\|_{S}\left\|\epsilon_{\beta}\right\|_{S} & =\sum_{\alpha}\left(s^{\alpha}\right)^{2}\left\|\epsilon_{\alpha}\right\|_{S}^{2} \\
\left.\sum_{i}\left(v^{i}\right)^{2} Q_{V}\left(e_{1}\right) \ldots \widehat{Q_{V}\left(e_{i}\right.}\right) \ldots Q_{V}\left(e_{n}\right) & =\sum_{i}\left(v^{i}\right)^{2} Q_{V}\left(e_{i}\right) .
\end{aligned}
$$

The above condition is equivalent to

$$
\left\|\epsilon_{\alpha}\right\|_{S}=0 \text { and }\left\|e_{1}\right\|_{V}^{2} \ldots\left\|\widehat{e_{i}}\right\|_{V}^{2} \ldots\left\|e_{n}\right\|_{V}^{2}=\left\|e_{i}\right\|_{V}^{2}
$$

but this is impossible.

\section{References}

[1] W. Królikowski, On Fueter-Hurwitz regular mappings, Dissertationes Math. 353 (1996).

[2] J. Ławrynowicz and J. Rembieliński, Pseudo-Euclidean Hurwitz pairs and generalized Fueter equations, in: Clifford algebras and their applications in mathematical physics, Proc., Canterbury 1985, J. S. R. Chisholm and A. K. Common (eds.), Reidel, Dordrecht, 1986, 39-48.

[3] -, - , On the composition of nondegenerate quadratic forms with an arbitrary index, Ann. Fac. Sci. Toulouse 10 (1989), 141-168.

[4] -, -, Pseudo-Euclidean Hurwitz pairs and the Katuża-Klein theories, J. Phys. A Math. Gen. 20 (1987), 5831-5848. 